\title{
First-year treatment costs among new initiators of topical prostaglandin analogs: pooled results
}

This article was published in the following Dove Press journal:

Clinical Ophthalmology

5 May 2010

Number of times this article has been viewed

\section{Jordana K Schmier' \\ David W Covert ${ }^{2}$}

'Managing Scientist, Exponent Inc., Alexandria, VA, USA; ${ }^{2}$ Associate Director, Health Economics, Alcon Research Ltd., Ft. Worth, TX, USA
Correspondence: Jordana K Schmier I 800 Diagonal Rd, Suite 300,

Alexandria,VA 223।4, USA

$\mathrm{Tel}+$ I 57| 227 724 |

Fax + I 57| 2277299

Email jschmier@exponent.com
Objective: To estimate first-year treatment costs among new initiators of topical prostaglandin analogs in a managed care population.

Research design and methods: A model was developed to estimate first-year medical costs. Model inputs were based on weighted results from three previous studies. Treatment patterns were derived from a claims database analysis. Published studies were used to estimate visit-related resource use. Costs were obtained from standard sources.

Results: Across studies, 27,809 patients met study criteria, 44.2\% of whom remained on their index therapy for 12 months. Adjunctive therapy was needed in $22.5 \%, 18.5 \%$, and $11.9 \%$ of bimatoprost, latanoprost, and benzalkonium chloride (BAK)-free travoprost patients, respectively. Median days to initiating adjunctive therapy were 64, 67, and 127 for bimatoprost, latanoprost, and BAK-free travoprost patients. Estimated first-year medical costs were $\$ 1,945, \$ 1,803$, and $\$ 1,730$ for patients initiating therapy with bimatoprost, latanoprost, and BAK-free travoprost. Findings were consistent through sensitivity analysis.

Conclusions: A BAK-free prostaglandin analog may permit longer duration of monotherapy and be associated with lower first-year treatment costs. Use of a claims database and the selection of new initiators of prostaglandin analogs limit the ability to project findings to all glaucoma patients.

Keywords: costs and cost analysis, drug therapy, combination, glaucoma, prostaglandin analogs

\section{Introduction}

Across studies of treatments for primary open-angle glaucoma (POAG), it is clear that there are many challenges to achieving and maintaining maximum reduction of intraocular pressure (IOP) and delaying disease progression. One challenge is increasingly complex regimens, known to be problematic for adherence in glaucoma treatment and across all chronic diseases. ${ }^{1-3}$ Proper instillation of eyedrops also cannot be assumed..$^{2,45}$ Another is the presence of adverse events, such as the development of ocular surface disease (OSD), ${ }^{3}$ that can affect adherence. ${ }^{6}$ In fact, approximately half of glaucoma patients experience some signs and/or symptoms of OSD in at least one eye. ${ }^{7,8}$

Given these impediments to adherence, the logical treatment should be a monotherapy with once daily dosing and a low likelihood of adverse events. However, even highly effective glaucoma therapies may not be sufficient to achieve adequate IOP reduction..$^{9-11}$ Prostaglandin analogs, which are recommended as first-line therapy for POAG by the American Academy of Ophthalmology, ${ }^{12}$ often require the use of 
adjunctive therapies, increasing exposure to preservatives commonly found in ophthalmic solutions. This increased exposure may decrease the success of filtration surgery if needed to treat more severe disease. ${ }^{13}$ A key question is whether there are differences among these recommended therapies, and a series of studies suggests that there may be one differentiating factor.

Of the three topical prostaglandin analogs currently approved and available in the United States, only travoprost is available without the preservative BAK. This newer product, BAK-free travoprost, is comparable in concentration to the conventional travoprost (including BAK) and has demonstrated equivalent IOP-lowering efficacy compared to travoprost with BAK. ${ }^{14}$ Furthermore, additional studies suggest that transitioning patients from a BAK-containing prostaglandin analog to BAK-free travoprost may improve ocular surface health, as indicated by signs and symptoms of OSD. ${ }^{15-21}$ Three studies explored the impact on use and type of adjunctive therapy and annual costs for patients initiating glaucoma therapy with prostaglandin analogs. ${ }^{22-24}$ Each study used the same inclusion criteria and identified patients with a prescription for a topical prostaglandin analog during a six-month period who had no glaucoma therapy claims in the six months prior and had at least 12 months of data available after the initial prostaglandin analog claim. Thus the analyses are comparable, although costs were updated to current values at the time each study was conducted.

The first study in the series ${ }^{24}$ compared treatment patterns and estimated annual costs of patients newly initiating therapy with one of three prostaglandin analogs: bimatoprost, latanoprost, or the BAK-free travoprost. Significantly fewer patients using BAK-free travoprost required adjunctive therapy compared to the other treatments, and the duration of monotherapy was twice as long for BAK-free travoprost (109 days) compared with the shortest duration of monotherapy identified (bimatoprost, 53 days). In addition, costs were approximately $11 \%$ lower for patients initiating therapy with BAK-free travoprost $(\$ 1,160)$ compared with the comparator with the highest first-year costs (bimatoprost, \$1,294). The second study in the series ${ }^{23}$ also found BAK-free travoprost associated with rates of adjunctive therapy use of less than half of comparators $(8.9 \%$ vs $16.5 \%$ for latanoprost and $20.7 \%$ for bimatoprost) and duration of monotherapy significantly lower than comparators (158.5 days vs 69.5 days for bimatoprost and 67.0 days for latanoprost). The differential in costs remained approximately $11 \%$, with first-year costs for BAK-free travoprost estimated to be $\$ 1,307$ compared with the highest cost of $\$ 1,457$ for bimatoprost. Finally, the third study in the series ${ }^{22}$ found that adjunctive intraocular pressure lowering therapy was needed in $23.6 \%, 18.5 \%$, and $13.3 \%$ of bimatoprost, latanoprost, and BAK-free travoprost patients, respectively. Median numbers of days to the first prescription filled for adjunctive therapy (if required) were 72.5, 74.0, and 125.0 for patients initiating on bimatoprost, latanoprost, and BAK-free travoprost. Total estimated first-year costs were $\$ 1,973, \$ 1,807$, and $\$ 1,739$ for patients initiating therapy with bimatoprost, latanoprost, and BAK-free travoprost.

The present study pooled data across all three studies, looking at 18 months of data on FDA-approved prostaglandin analogs.

\section{Methods}

We used three primary sources of input into the economic model. We first conducted multiple retrospective cohort studies, over time, using a prescription benefits database. ${ }^{22-24}$ Data from these analyses were pooled and were used to identify the population of patients receiving prostaglandin analogs and to explore patterns of use of adjunctive therapies. Second, we reviewed published studies identified through a literature review to estimate the components of a typical outpatient visit (initial evaluation, diagnostics tests, and follow-up care). Finally, we consulted standard cost sources to provide costs for each resource (initial treatments, adjunctive therapies, and visits) identified.

\section{Database analysis: study population and use of adjunctive therapies}

The patients described in the retrospective cohort analysis were receiving prescription benefits and were included in a prescription claims database of a large pharmaceutical benefits manager (PBM). This PBM serves more than 75 million plan participants across the United States. All data were de-identified in accordance with Protected Health Information standards under the Health Information Portability and Accountability Act so that no individually identifiable information was included in the study database. Therefore, review by an institutional review board was not required.

The study cohort included patients who first initiated therapy with one of three prostaglandin analog products (bimatoprost, latanoprost, or BAK-free travoprost) between November 1, 2006 and April 30, 2008. To qualify for each of the previous three studies, patients had to have more than one prescription claim of their index prostaglandin analog and six months of prior claims data in which there were no glaucoma therapy claims of any class. Glaucoma therapy claims were 
defined by therapeutic class plus generic code number $(\mathrm{GCN})$ codes. GCNs are a system of unique numbers assigned by drug pricing service First DataBank to medications according to strength, formulation, route of administration, and size. The study population was further defined by requiring patients to have at least 12 months of uninterrupted prescription refills of the index prostaglandin analog following the initial prescription. This was operationalized as not having prescriptions for the other prostaglandin analogs during the year and having at least one prescription for the index prostaglandin analog in the fourth quarter of the follow-up period. Patients were required to be continuously enrolled during the 18-month period (six months prior to the first prostaglandin claim and the 12 months of follow-up), according to the PBM's enrollment files. Patients who did not meet these requirements (sufficient prior claims data, uninterrupted use, and use of the index prostaglandin analog during the fourth quarter of observation) were excluded from the study database. We considered that patients added an adjunctive medication, defined by therapeutic class plus GCN codes, if there was a sequential and subsequent refill of an adjunctive agent in the presence of continued refills for the index agent.

\section{Literature review: resource use and cost inputs}

The American Academy of Ophthalmology Preferred Practice Patterns for glaucoma suggest that follow-up care should be based on achievement of target IOP and the amount of disease progression, ${ }^{12}$ neither of which was available in the prescription database used for this study. Therefore, the base case analysis uses resource rates from a survey-based study ${ }^{25}$ while sensitivity analyses explore visits as recommended by other studies and guidelines. ${ }^{12,26}$ Since all patients in the model are assumed to undergo the same procedures and tests at their visits regardless of the prostaglandin analog they receive, the relatively small differences across studies in terms of the components of each visit have minimal impact on study findings.

At the initial visit, patients are assumed to have a level four (comprehensive) evaluation (CPT 92004). Follow-up visits are assumed to be level two (intermediate) visits (CPT 92012), with three follow-up visits during the year (likely but not necessarily at 30 and 90 days following initiation of the index therapy, and at 12 months). Two additional follow-up visits are assumed to be associated with the initiation and monitoring of adjunctive medication among patients for whom it is prescribed. Thus patients who stay on monotherapy are assumed to have one comprehensive visit and three intermediate visits while those who require adjunctive therapy had one comprehensive visit and five intermediate visits. As the model only considered one year of treatment costs, it was not essential when in the year these visits occurred (ie, discounting based on timing was not necessary); only the total number and type were relevant. Table 1 presents the procedures and diagnostic tests that comprise each visit as well as the costs used for each.

We used average wholesale price (AWP) for 2009 as the basis of prescription costs. ${ }^{27}$ The range of published AWPs for the prostaglandin analogs was fairly narrow ( $\$ 80.53$ to \$82.67). ${ }^{27}$ For the prostaglandin analogs, the midpoint cost for the agents is used in the model because AWP is the best publicly available estimate for the analysis. Further, the number of prescriptions for prostaglandin analogs was expressed as

Table I Unit costs

\begin{tabular}{|c|c|c|}
\hline Resource & Description/size & Cost \\
\hline Initial visit & $\begin{array}{l}\text { CPT } 92004 \text { (comprehensive, new patient) plus weighted costs } \\
\text { for visual field exam (92082), gonioscopy (92020), evaluation } \\
\text { of optic disc (92135), optic nerve head photograph (92235), } \\
\text { and fundus evaluation (92250) per Fremont et a }\left.\right|^{25}\end{array}$ & $\$ 582.61^{28}$ \\
\hline Follow-up visit & $\begin{array}{l}\text { CPT } 92012 \text { (intermediate, established patient) and visual acuity } \\
\text { exam ( } 99173) \text { per Fremont et } \mathrm{al}^{25}\end{array}$ & $\$ 117.68^{28}$ \\
\hline Prostaglandin analog & $2.5 \mathrm{~mL}$ & $\$ 81.60^{27}$ \\
\hline $\begin{array}{l}\text { Fixed-combination dorzolamide } \\
2.0 \% / \text { timolol } 0.5 \%\end{array}$ & $5 \mathrm{~mL}$ & $\$ 130.80^{27}$ \\
\hline Brimonidine $0.1 \%$ & $5 \mathrm{~mL}$ & $\$ 62.80^{27}$ \\
\hline Brinzolamide $1.0 \%$ & $10 \mathrm{~mL}$ & $\$ 91.38^{27}$ \\
\hline Timolol $0.25 \%$ & $5 \mathrm{~mL}$ & $\$ 17.93^{27}$ \\
\hline Other & $5 \mathrm{~mL}$ & $\begin{array}{l}\$ 75.73 \\
\text { (assumption) }\end{array}$ \\
\hline
\end{tabular}

Note: Costs are expressed in 2009 US\$. 
$2.5 \mathrm{~mL}$ size bottle equivalents, as this is the most common size found in the claims database and represents approximately a 30-45 day supply when used per label in both eyes. For example, a $5 \mathrm{~mL}$ bottle was treated as two $2.5 \mathrm{~mL}$ bottles. The AWPs for adjunctive therapies were also reviewed in the Red Book. ${ }^{27}$ For the base case of the model, branded products were used, but sensitivity analyses explored lower costs. Days of use per bottle were based on data from these studies. The number of bottles required was rounded to the nearest tenth. Medical charges were estimated using the 75th percentile of physicians' fees from a published benchmark. ${ }^{28}$ All the costs used in the model are presented in Table 1.

\section{Analysis}

The analysis identified patients by the prostaglandin prescription they first filled (ie, the "index medication") during the study period. Gender was compared using a chi-square test and age was compared using analysis of variance (ANOVA) with Bonferroni's correction for multiple comparisons. The number of refills per year of each prostaglandin was calculated to use as an input to the economic model. Median and mean number of days to initiation of adjunctive therapy were calculated for each cohort, with the distribution of number of days examined to determine which measure to use in the model (ie, mean if the days were normally distributed, median if they were not). The median numbers of days until patients added adjunctive therapies were compared using the Wilcoxon rank sum test; mean times were compared using ANOVA. Statistical comparisons were conducted in SAS (v9.2; SAS Institute, Inc., Cary, NC).
Sensitivity analyses modified clinical and cost input parameters and explored the impact of assumptions on model findings. Two clinical inputs were varied. First, the proportion of patients remaining on monotherapy for the entire year was changed to the lowest and highest values among the initial treatments, that is, the proportion of patients remaining on monotherapy was changed to $77.5 \%$ (the base case value for bimatoprost) and then to $88.1 \%$ (the base case value for BAK-free travoprost) for all treatments. Second, the days to initiating adjunctive therapy was varied in two ways: a) median number of days to the addition of adjunctive medication was changed to the lowest and highest values among the treatments and $b$ ) the mean number of days was substituted for the median. A number of cost-related variables were also subjected to sensitivity analyses. The costs of treatments were varied by assuming that the least and most expensive adjunctive therapies were the only therapies used. Sensitivity analysis was also conducted on the cost of visits, using resource use estimates from other published sources. ${ }^{12,26} \mathrm{~A}$ minimum value for frequency of visits and resource use was estimated from the American Academy of Ophthalmology Preferred Practice Patterns ${ }^{12}$ and assumed that patients were meeting IOP targets; the maximum value assumed that patients were not meeting IOP targets and that there was progression of disease. In addition, the 50th percentile of physicians' fees was substituted for the 75 th percentile.

\section{Results}

Figure 1 provides a flow chart of the patient selection process for the retrospective cohort analysis. Of more than

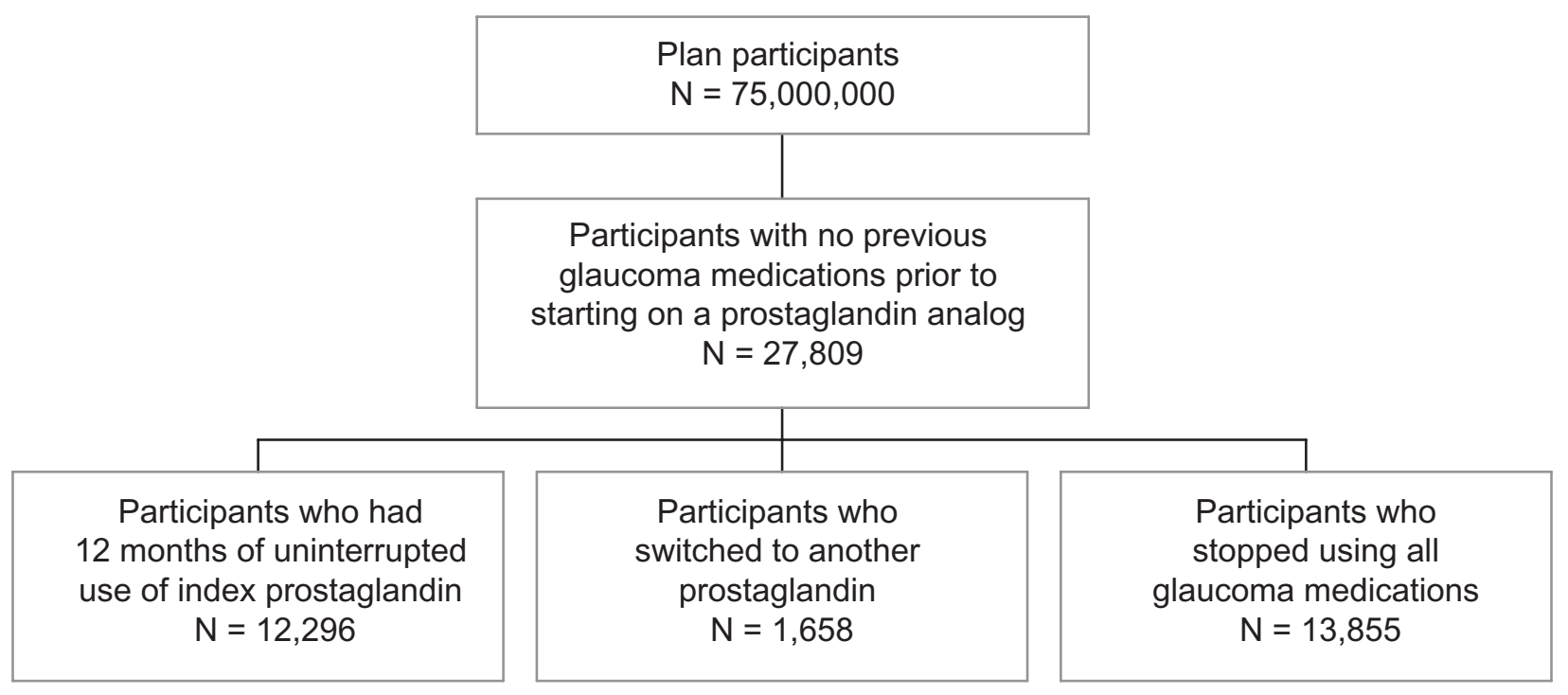

Figure I Patient selection. 
75 million plan participants, 27,809 initiated treatment with a prostaglandin analog. Approximately $6 \%$ of these patients switched from their index prostaglandin analog to another glaucoma medication and about $50 \%$ of the patients stopped taking all glaucoma medications within the 12-month period. Of those switching, the primary alternative therapies were another prostaglandin analog, beta-blocker, or fixed combination therapy. The analysis examined only those newly initiating patients who remained on their initial prostaglandin analog therapy for one year, which represents approximately $44 \%$ of the glaucoma patients newly initiating with prostaglandin analogs identified from the database.

Demographic characteristics of the newly initiating patients are presented in Table 2. More than half (55.4\%) of the patients were women $(P<0.01)$. Mean age ranged from 63 to 65 years and was significantly different across groups $(P<0.0001$, with BAK-free travoprost significantly different by less than two years from the other prostaglandin analogs).

The mean number of bottles per year of each prostaglandin (expressed as $2.5 \mathrm{~mL}$ bottle equivalents) was calculated, based on weighting findings from previous studies (8.7 for BAK-free travoprost, 9.0 for latanoprost, and 10.2 for bimatoprost), and was used to calculate costs for prostaglandin analogs. In the bimatoprost, latanoprost, and BAK-free travoprost treatment groups, $22.5 \%, 18.5 \%$, and $11.9 \%$, respectively, initiated adjunctive therapy during the course of the year at significantly different proportions $(P<0.0001$, see Table 3). Patients on BAK-free travoprost were able to continue without adjunctive therapy longer than patients treated with other prostaglandins. Figure 2 shows that the median numbers of days until patients added adjunctive therapies were 127 days for patients initiating therapy with BAK-free travoprost, 64 days for bimatoprost, and 67 days for latanoprost ( $P=0.0004$, Wilcoxon rank sum test). The mean numbers of days to initiating adjunctive therapy were 144.9 days in the BAK-free travoprost group, 107.5 for patients receiving bimatoprost, and 105.4 for patients

Table 2 Demographic characteristics

\begin{tabular}{llll}
\hline Characteristic & $\begin{array}{l}\text { BAK-free } \\
\text { travoprost }\end{array}$ & Bimatoprost & Latanoprost \\
\hline $\mathrm{N}$ & 3,404 & 6,184 & 18,221 \\
Age, mean \pm & $62.9 \pm 13.3$ & $64.5 \pm 14.2$ & $64.1 \pm 14.4$ \\
standard deviation & & & \\
Gender, \% female $^{* * *}$ & $54.6 \%$ & $53.7 \%$ & $56.2 \%$ \\
\hline
\end{tabular}

Notes: ${ }^{*} P<0.0001$ across treatment groups (BAK-free travoprost significantly different from bimatoprost and latanoprost). ${ }^{* *} P<0.0$ I. receiving latanoprost. Mean time to initiating adjunctive therapy was also significantly different across the cohorts $(P=0.0002$, ANOVA $)$.

Figure 3 presents the primary findings from the analysis. Estimated annual costs for patients initiating therapy with BAK-free travoprost were lowest $(\$ 1,730$; $\$ 964$ medical and $\$ 766$ pharmacy), with increasing annual costs for latanoprost (\$1,803; \$979 medical and \$824 pharmacy), and bimatoprost (\$1,945; \$989 medical and $\$ 956$ pharmacy). For all agents, visits comprised at least $50 \%$ of the total annual cost (50.8\% for bimatoprost to $55.7 \%$ for BAK-free travoprost).

Results of sensitivity analyses are shown in Table 4. Findings remained consistent across univariate sensitivity analyses, with BAK-free travoprost having the lowest annual cost and bimatoprost having the highest cost in all scenarios. In the base case, the range of one-year costs across index medications was $12 \%$; in univariate sensitivity analyses, it ranged from $8 \%$ to $16 \%$. The single most influential assumption was that all adjunctive therapy received was the most costly agent available. Bivariate sensitivity analyses resulted in differences in the range of 3\% (highest rate of continued monotherapy and visit components based on AAO targets not met) $)^{12}$ to $18 \%$ across index medications (most costly adjunctive therapy and visit components based on Quigley estimates). ${ }^{26}$ In all cases, BAK-free travoprost remained the least costly option and bimatoprost the most costly option.

\section{Discussion}

This analysis adds to a growing body of literature finding consistent differences in treatment patterns and costs for glaucoma patients initiating treatment with prostaglandin analogs with varying rates of adjunctive therapy use. Over a period of 18 months after initial availability of BAK-free travoprost, patients initiating glaucoma treatment with this medication remained on monotherapy in greater proportions and for a longer duration compared to other prostaglandin analogs.

The early studies in this series included fewer patients as it takes time for patients to be transitioned to a new formulation of an existing product. Although significant findings were detected in each study, there are benefits to pooling the data. Small variations are likely washed out, and the variance of continuous variables is narrower. For example, while the standard deviations of the mean time to adjunctive therapy remained large, they were considerably tighter in the pooled analysis. Using pooled results, with a larger population as the base for the model input parameters, may also suggest that the results are more generalizable. 
Table 3 Treatment patterns during follow-up period

\begin{tabular}{llll}
\hline Treatment pattern & $\begin{array}{l}\text { BAK-free } \\
\text { travoprost }\end{array}$ & Bimatoprost & Latanoprost \\
\hline Number remaining on index therapy (N) & 1,210 & 2,644 & 8,442 \\
Remained on monotherapy (\%) & $88.1 \%$ & $77.5 \%$ & $81.5 \%$ \\
Required adjunctive therapy (\%) & $11.9 \%$ & $22.5 \%$ & $18.5 \%$ \\
Type of adjunctive therapy: & & & $23.0 \%$ \\
$\alpha_{2}$-adrenergic receptor agonist (\%) & $29.4 \%$ & $27.1 \%$ & $40.1 \%$ \\
Beta-blocker (\%) & $28.9 \%$ & $32.1 \%$ & $16.0 \%$ \\
Carbonic anhydrase inhibitor (\%) & $17.8 \%$ & $15.2 \%$ & $17.3 \%$ \\
Fixed-combination beta-blocker/carbonic & $20.6 \%$ & $22.1 \%$ & $3.5 \%$ \\
anhydrase inhibitor (\%) & & & $3.5 \%$ \\
$\quad$ Other adjunctive therapy (\%) & $3.3 \%$ & & \\
\hline
\end{tabular}

Note: ${ }^{*} P<0.0001$ (chi-square).

The use of a large claims database offers an important strength in terms of sample size. However, there are also disadvantages to the use of a pharmaceutical claims database without additional clinical input in terms of characterizing patients and/or prescribers. ${ }^{29}$ Patients are not randomized to treatment, although age and gender of the population reflected that of the general glaucoma population ${ }^{30}$ and varied little across treatment groups. The reasons for adding adjunctive therapy, switching, or discontinuing therapies cannot be surmised from a claims database alone. In addition, claims databases report on prescriptions filled, but cannot be used as a definitive statement of adherence to therapy. ${ }^{31-34}$ Also affecting adherence and cost estimates is that there is no way to account for product samples, which one study identified as being received by $20 \%$ of patients. ${ }^{29}$ We assumed that the use of samples would be similar across products studied in a database of this size. The population of prescribers may not accurately reflect national patterns, which could misrepresent prescribing patterns. There are no data on the number of prescribers whose patients appear in the database, but as the data were from a large PBM, it is likely that there is a wide distribution of prescribers and practices. Finally, claims databases may contain coding biases or errors, although there is no reason to believe that these errors would be different across index treatments; in addition, the claims in this database were reviewed and adjudicated before the database was prepared for this analysis.

Beyond the limitations of a pharmaceutical claims database, other assumptions may also have affected our study findings. The study attempted to identify only patients who were new to therapy, although even a criterion as simple as this is difficult to implement. ${ }^{29}$ Also, based on the study methodology, patients could have had poor adherence but still have been included; patients in the cohort analysis were required to have a minimum of two prescriptions for the same prostaglandin analog filled during the follow-up period, but no attempt was

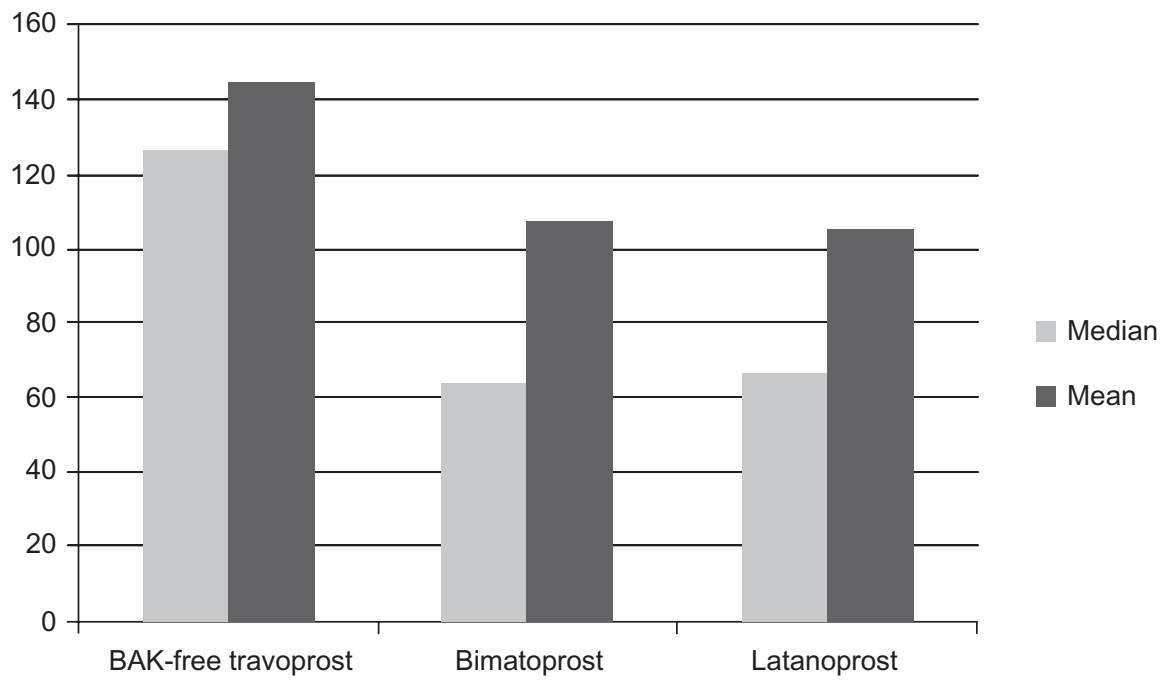

Figure 2 Days to initiating adjunctive therapy. 


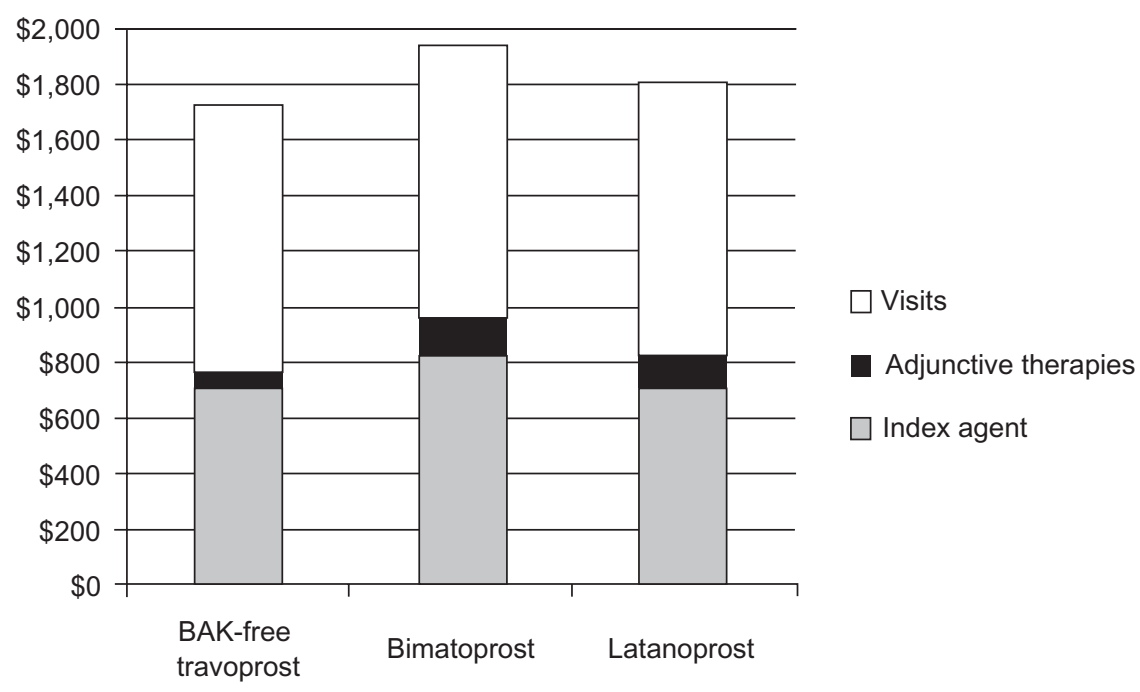

Figure 3 Annual average cost per patient by category.

made to assess medication possession ratio or to evaluate persistence with therapy over time. The subset of patients identified through this analysis may not be representative of glaucoma patients in other aspects, although the nature of a prescription claims database does not allow detailed exploration. For example, we could not detect other comorbid conditions, although it may have been possible to make some assumptions based on a thorough evaluation of all prescription medications taken by each patient. All assumptions about costs and resource utilization were explored through sensitivity analysis.
As with any economic model, findings are dependent on the strength of the assumptions. We have made every attempt to be transparent about the source of the input parameters, to use data from public and/or peer-reviewed sources, and we conducted thorough sensitivity analyses on the impact of these parameters. There will always remain uncertainty inherent in models, but the difficulty of obtaining complete data on individual patients is often insurmountable. For example, data vendors tend to provide standardized costs rather than actual costs and even complete electronic

Table 4 Univariate sensitivity analyses

\begin{tabular}{|c|c|c|c|}
\hline $\begin{array}{l}\text { Parameter changed value } \\
\text { to which it was changed }\end{array}$ & BAK-free travoprost & Bimatoprost & Latanoprost \\
\hline Base case & $\$ 1,730$ & $\$ 1,945$ & $\$ 1,803$ \\
\hline \multicolumn{4}{|l|}{ Patients on monotherapy (\%) } \\
\hline Decreased to $77.5 \%$ for all & $\$ 1,803$ & $\$ 1,945^{*}$ & $\$ 1,833$ \\
\hline Increased to $88.1 \%$ for all & $\$ 1,730 *$ & $\$ 1,86 \mid$ & $\$ 1,754$ \\
\hline \multicolumn{4}{|l|}{ Days to adjunctive therapy } \\
\hline Mean days to adjunctive therapy & $\$ 1,726$ & $\$ 1,927$ & $\$ 1,791$ \\
\hline Decreased to 64 days for all & $\$ 1,744$ & $\$ 1,945^{*}$ & $\$ 1,804$ \\
\hline Increased to 144.9 days for all & $\$ 1,726$ & $\$ 1,911$ & $\$ 1,779$ \\
\hline \multicolumn{4}{|l|}{ Cost of therapies } \\
\hline Changed to least costly adjunctive therapy & $\$ 1,690$ & $\$ 1,855$ & $\$ 1,737$ \\
\hline Changed to most costly adjunctive therapy & $\$ 1,780$ & $\$ 2,067$ & $\$ 1,909$ \\
\hline \multicolumn{4}{|l|}{ Cost of visits - alternative practice } \\
\hline \multicolumn{4}{|l|}{ patterns and costs } \\
\hline Based on Quigley et $\mathrm{a}^{26}$ & $\$ 1,546$ & $\$ 1,761$ & $\$ 1,620$ \\
\hline \multicolumn{4}{|c|}{ Based on American Academy of Ophthalmology'12 } \\
\hline Targets met (minimum) & $\$ 2,022$ & $\$ 2,237$ & $\$ 2,095$ \\
\hline Targets not met (maximum) & $\$ 4,269$ & $\$ 4,631$ & $\$ 4,434$ \\
\hline Based on 75th percentile UCR & $\$ 2,021$ & $\$ 2,244$ & $\$ 2,100$ \\
\hline \multicolumn{4}{|l|}{ Cost of prostaglandin analogs } \\
\hline Actual costs & $\$ 1,724$ & $\$ 1,937$ & $\$ 1,794$ \\
\hline Round up to nearest whole number & $\$ 1,734$ & $\$ 1,951$ & $\$ 1,810$ \\
\hline
\end{tabular}

Note: *No change from base case. 
health records cannot consistently answer why patients discontinue treatment. Many studies report high rates of discontinuation with glaucoma treatment but provide no definitive answers, ${ }^{35-37}$ even when the interesting question of restarts during the observation period is considered. ${ }^{38}$

These findings showed that prostaglandin analogs with a greater proportion of patients remaining on monotherapy and a longer time to initiation of adjunctive agents had lower first-year direct medical costs from a third party payer's perspective. Additionally, while our resource use estimates assumed that follow-up visits to evaluate adjunctive therapies would be coded as intermediate visits, up-coding (ie, coding these visits as comprehensive) could occur and this would further differentiate products by rates of adjunctive therapy use. Accordingly, costs from the patient's perspective (eg, co-pays for visits and prescriptions) would also be lower with longer duration of monotherapy. Interestingly, there were no remarkable differences in the distribution of types of adjunctive therapies across treatment groups. Further study is needed to identify to what extent tolerability, specifically the absence of BAK, contributes to use of adjunctive medications either to supplement glaucoma treatment or to treat resultant co-morbidities such as dry eye or ocular surface disease.

There are important follow-up questions to these results. Why are there differences in rates of adjunctive therapy? Are there also differences in disease progression or the rate of surgical interventions? What other patient characteristics might affect use of adjunctive therapy, adherence with therapy, or rates of disease progression? Unfortunately, the use of pharmaceutical databases unaided by medical claims information leaves these questions unanswered. We can speculate that patients who do not experience OSD may be more likely to adhere to their prostaglandin treatment for longer periods of time, as has been shown with other studies of BAK-free travoprost, ${ }^{14,16,39}$ but other variables may be relevant. We are currently undertaking an analysis of a comprehensive health insurance claims database over a two-year time horizon in an effort to explore some of these questions.

\section{Disclosures}

Author JKS is an employee of Exponent, which received funding from Alcon to conduct this study. Author DWC is an employee of Alcon Research Ltd.

\section{References}

1. Yeaw J, Benner JS, Walt JG, Sian S, Smith DB. Comparing adherence and persistence across 6 chronic medication classes. J Manag Care Pharm. 2009;15(9):728-740.
2. Robin AL, Novack GD, Covert DW, Crockett RS, Marcic TS. Adherence in glaucoma: objective measurements of once-daily and adjunctive medication use. Am J Ophthalmol. 2007;144(4):533-540.

3. Robin AL, Covert D. Does adjunctive glaucoma therapy affect adherence to the initial primary therapy? Ophthalmology. 2005;112(5):863-868.

4. Stone JL, Robin AL, Novack GD, Covert DW, Cagle GD. An objective evaluation of eyedrop instillation in patients with glaucoma. Arch Ophthalmol. 2009;127(6):732-736.

5. Okeke CO, Quigley HA, Jampel HD, et al. Adherence with topical glaucoma medication monitored electronically the Travatan Dosing Aid study. Ophthalmology. 2009;116(2):191-199.

6. Friedman DS, Hahn SR, Gelb L, et al. Doctor-patient communication, health-related beliefs, and adherence in glaucoma results from the Glaucoma Adherence and Persistency Study. Ophthalmology. 2008;115(8):1320-1327.

7. Leung EW, Medeiros FA, Weinreb RN. Prevalence of ocular surface disease in glaucoma patients. J Glaucoma. 2008;17(5):350-355.

8. Fechtner RD, Godfrey DG, Budenz D, Stewart JA, Stewart WC, Jasek MC. Prevalence of ocular surface complaints in glaucoma patients using topical intraocular pressure-lowering medications. Cornea. 2010; Apr 9. [Epub ahead of print]. doi:10.1097/ICO.0b013e3181c325b2.

9. Sit AJ, Weinreb RN, Crowston JG, Kripke DF, Liu JH. Sustained effect of travoprost on diurnal and nocturnal intraocular pressure. Am J Ophthalmol. 2006;141(6):1131-1133.

10. Costagliola C, Del Prete A, Verolino M, et al. Effect of $0.005 \%$ latanoprost once daily on intraocular pressure in glaucomatous patients not adequately controlled by beta-blockers twice daily: a 3-year follow-up. Experience and incidence of side effects in a prospective study on 76 patients. Graefes Arch Clin Exp Ophthalmol. 2002;240(5):379-386.

11. Netland PA, Landry T, Sullivan EK, et al. Travoprost compared with latanoprost and timolol in patients with open-angle glaucoma or ocular hypertension. Am J Ophthalmol. 2001;132(4):472-484.

12. American Academy of Ophthalmology Glaucoma Panel, Preferred Practice Patterns Committee. Primary Open-Angle Glaucoma. Limited Revision. San Francisco, CA: American Academy of Ophthalmology; 2003.

13. Broadway DC, Grierson I, O'Brien C, Hitchings RA. Adverse effects of topical antiglaucoma medication. II. The outcome of filtration surgery. Arch Ophthalmol. 1994;112(11):1446-1454.

14. Lewis RA, Katz GJ, Weiss MJ, et al. Travoprost $0.004 \%$ with and without benzalkonium chloride: a comparison of safety and efficacy. J Glaucoma. 2007;16(1):98-103.

15. Baudouin C. Detrimental effect of preservatives in eyedrops: implications for the treatment of glaucoma. Acta Ophthalmol. 2008;86(7): 716-726.

16. Henry JC, Peace JH, Stewart JA, Stewart WC. Efficacy, safety, and improved tolerability of travoprost BAK-free ophthalmic solution compared with prior prostaglandin therapy. Clin Ophthalmol. 2008;2: 613-621.

17. Kahook MY, Noecker RJ. Comparison of corneal and conjunctival changes after dosing of travoprost preserved with sofZia, latanoprost with $0.02 \%$ benzalkonium chloride, and preservative-free artificial tears. Cornea. 2008;27(3):339-343.

18. Horsley MB, Kahook MY. Effects of prostaglandin analog therapy on the ocular surface of glaucoma patients. Clin Ophthalmol. 2009;3:291-295.

19. Cha SH, Lee JS, Oum BS, Kim CD. Corneal epithelial cellular dysfunction from benzalkonium chloride (BAC) in vitro. Clin Experiment Ophthalmol. 2004;32(2):180-184.

20. Whitson JT, Cavanagh HD, Lakshman N, Petroll WM. Assessment of corneal epithelial integrity after acute exposure to ocular hypotensive agents preserved with and without benzalkonium chloride. Adv Ther. 2006;23(5):663-671.

21. Yee RW, Norcom EG, Zhao XC. Comparison of the relative toxicity of travoprost $0.004 \%$ without benzalkonium chloride and latanoprost $0.005 \%$ in an immortalized human cornea epithelial cell culture system. Adv Ther. 2006;23(4):511-519. 
22. Schmier JK, Covert DW, Robin AL. First-year treatment costs among new initators of topical prostaglandin analogs. Clin Opthalmol. 2009;3:637-644.

23. Schmier JK, Covert DW, Robin AL. First-year treatment costs among new initiators of topical prostaglandin analogs. Clin Ophthalmol. 2009;3:637-644

24. Schmier JK, Covert DW, Robin AL. First-year treatment patterns among new initiators of topical prostaglandin analogs. Curr Med Res Opin. 2009;25(4):851-858.

25. Fremont AM, Lee PP, Mangione CM, et al. Patterns of care for open-angle glaucoma in managed care. Arch Ophthalmol. 2003;121(6): 777-783.

26. Quigley HA, Friedman DS, Hahn SR. Evaluation of practice patterns for the care of open-angle glaucoma compared with claims data the Glaucoma Adherence and Persistency Study. Ophthalmology 2007;114(9):1599-1606.

27. Thomson Healthcare. Red Book Drug Topics. Montvale, NJ: Thomson Healthcare; 2009.

28. Physicians' Fee Reference: Comprehensive Fee Report: New Haven, CT: Yale Wasserman D.M.D. Medical Publishers; 2009.

29. Friedman DS, Quigley HA, Gelb L, et al. Using pharmacy claims data to study adherence to glaucoma medications: methodology and findings of the Glaucoma Adherence and Persistency Study (GAPS). Invest Ophthalmol Vis Sci. 2007;48(11):5052-5057.

30. Friedman DS, Wolfs RC, O'Colmain BJ, et al. Prevalence of open-angle glaucoma among adults in the United States. Arch Ophthalmol. 2004;122(4):532-538
31. Brown MM, Brown GC, Spaeth GL. Improper topical self-administration of ocular medication among patients with glaucoma. Can J Ophthalmol. 1984;19(1):2-5.

32. Ashburn FS Jr, Goldberg I, Kass MA. Compliance with ocular therapy. Surv Ophthalmol. 1980;24:237-248.

33. Kholdebarin R, Campbell RJ, Jin YP, Buys YM. Multicenter study of compliance and drop administration in glaucoma. Can J Ophthalmol. 2008;43:454-461.

34. Norell SE, Granstrom PA. Self-medication with pilocarpine among outpatients in a glaucoma clinic. $\mathrm{Br} J$ Ophthalmol. 1980;64:137-141

35. Vanelli M, Pedan A, Liu N, Hoar J, Messier D, Kiarsis K. The role of patient inexperience in medication discontinuation: a retrospective analysis of medication nonpersistence in seven chronic illnesses. Clin Ther. 2009;31(11):2628-2652.

36. Nordstrom BL, Friedman DS, Mozaffari E, Quigley HA, Walker AM. Persistence and adherence with topical glaucoma therapy. Am J Ophthalmol. 2005;140(4):598-606.

37. Shaya FT, Mullins CD, Wong W, Cho J. Discontinuation rates of topical glaucoma medications in a managed care population. Am J Manag Care. 2002;8 Suppl 10:S271-S277.

38. Schwartz GF, Platt R, Reardon G, Mychaskiw MA. Accounting for restart rates in evaluating persistence with ocular hypotensives. Ophthalmology. 2007;114(4):648-652.

39. Gross RL, Peace JH, Smith SE, et al. Duration of IOP reduction with travoprost BAK-free solution. J Glaucoma. 2008;17(3):217-222.
Clinical Ophthalmology

\section{Publish your work in this journal}

Clinical Ophthalmology is an international, peer-reviewed journal covering all subspecialties within ophthalmology. Key topics include: Optometry; Visual science; Pharmacology and drug therapy in eye diseases; Basic Sciences; Primary and Secondary eye care; Patient Safety and Quality of Care Improvements. This journal is indexed on

Submit your manuscript here: http://www.dovepress.com/clinical-ophthalmology-journal

\section{Dovepress}

PubMed Central and CAS, and is the official journal of The Society of Clinical Ophthalmology (SCO). The manuscript management system is completely online and includes a very quick and fair peer-review system, which is all easy to use. Visit http://www.dovepress.com/ testimonials.php to read real quotes from published authors. 